\title{
Diffuse Intramural Pseudodiverticulosis
}

Diffuse intramural pseudodiverticulosis of the esophagus is a rare benign condition. It was first described by Mendl et al. in 1960 (1), and since then, several cases have been reported. The endoscopic features of the condition have rarely been described. We report here a case detected incidentally in a 41 -year-old patient with alcoholic cirrhosis of the liver.

The patient was referred to our department on an emergency basis for mild hematemesis. Upper gastrointestinal endoscopy showed multiple tiny saccular spaces involving the whole length of the esophagus (Figure 1), most being located in the distal portion. The mucosa was macroscopically normal, and a small $(4 \mathrm{~mm}$ in diameter) peptic ulcer was observed at the gastroesophageal junction. A few days later, a barium esophagogram confirmed the endoscopic diagnosis (Figure 2). Esophageal manometry did not show any alteration. The patient was discharged one week later, with $20 \mathrm{mg}$ /day omeprazole therapy.

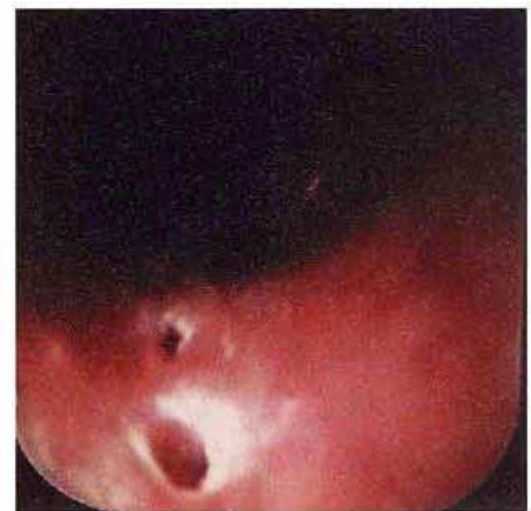

Figure 1: Endoscopic view of multiple tiny saccular spaces involving the wall of the esoph. agus. 


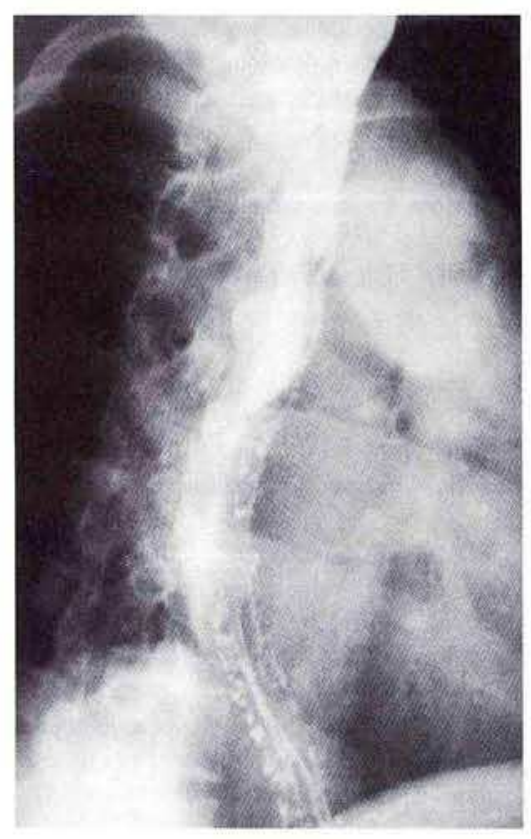

Figure 2: Barium esophagogram. Pseu dodiverticula can be seen in the distal portion of the esophagus.
The pathology of this anomaly consists of cystic dilations of the excretory ducts of the submucosal glands, and a mucocele can sometimes develop (2). A mild stenosis in the upper esophagus may be present. Candidiasis, chronic esophagitis, and motor abnormalities may be associated with this condition, but none of these was observed in our patient. No specific therapy was necessary in this patient, but when a stenosis is detected, a simple endoscopic dilation can quickly solve the problem.

F-X. Caroli-Bosc, M. Conio, B. Diaine, K. Arab, J.-F. Demarquay, P. Hastier, J.-P. Delmont

Dept. of Gastroenterology, Cimiez Hospital, University of Nice, Nice, France

\section{References}

1. Mendl K, Tanner CH, McKay J. Intramural diverticulosis of the oesophagus and Rokitansky - Aschoff sinuses of the gallbladder. Br J Radiol 1960; 33: 496-501.

2. Umlas J, Sakhuja R. The pathology of esophageal intramural pseudodiverticulosis. Am J Clin Pathol 1976; 65: 314-20.

Corresponding Author

F.-X. Caroli-Bosc, Service d'Hépato-Gastroentérologie Hôpital Universitaire de l'Archet

151 , route Saint-Antoine de Ginestière

06202 Nice Cedex 3, France, Fax: + 33-92036134 